\title{
Genetic diversity and relationship of Halla horse based on polymorphisms in microsatellites
}

\author{
Ji Su Jung ${ }^{1,2}$, Jiyeon Seong ${ }^{3}$, Gwang Hyeon Lee ${ }^{4}$, Yesong Kim ${ }^{4}$, Je Hyun An ${ }^{4}$, Ji Hye Yun ${ }^{4}$ and \\ Hong Sik Kong ${ }^{1,4,5, *}$ \\ ${ }^{1}$ Hankyong and Genetics, Anseong 17579, Korea \\ ${ }^{2}$ The Graduate School, Department of Animal Life and Environment Science, Hankyong National University, Anseong \\ 17579 , Korea \\ ${ }^{3}$ Genomic Informatics Center, Hankyong National University, Anseong 17579, Korea \\ ${ }^{4}$ Department of Applied Biotechnology, The Graduate School of Hankyong National University, Anseong 17579, Korea \\ ${ }^{5}$ Gyeonggi Regional Research Center, Hankyong National University, Anseong 17579, Korea
}

Received March 8, 2021

Revised April 24, 2021

Accepted May 12, 2021

\section{*Correspondence \\ Hong Sik Kong \\ E-mail: kebinkhs@hknu.ac.kr}

ORCID

https://orcid.org/000-0003-1144-016X

\begin{abstract}
Halla horse is crossbreed between Jeju and Thoroughbred horses and is used for riding, racing and meat production. Thus, molecular genetic studies are needed to establish and preserve the industrially valuable Halla horses. This study aimed to analyses the genetic diversity and population structure through 12 microsatellite (MS) markers for Halla and putatively related 3 breeds (Jeju, Mongolian and Thoroughbred horses). On average, the number of alleles, observed heterozygosity $\left(\mathrm{H}_{\mathrm{obs}}\right)$, expected heterozygosity $\left(\mathrm{H}_{\text {exp }}\right)$, and polymorphic information content (PIC) among all horses were $10,0.767,0.799$, and 0.771 , respectively. Neighbor-joining tree and STRUCTURE analysis showed that Halla horses were between Thoroughbred and Jeju horses, tend to more influenced by Thoroughbred horses. Therefore, these results could be considered for use as the basic genetic breed relationships resource among the horse breeds (Jeju, Mongolian, and Thoroughbred horses) related to the origins of the Halla horse.
\end{abstract}

Keywords: Halla horse, horse, genetic diversity, relationship, Microsatellite marker

\section{INTRODUCTION}

In the past, horses were used to the development of means of transport and communication and spread of agricultural machinery. However, the quality of human life improved, the range of uses of horses changed for purposes related to cultural life, such as leisure activities and sightseeing (Seo et al., 2016).

According to 2016 report, about 26,000 horses are breeding in Korea and $57.2 \%$ of them are breeding in Jeju island (Seo et al., 2016). The horses breeding in Jeju is- land are subdivided into Halla horses (75.1\%), Jeju horses (6.1\%) and Thoroughbred horses (18.8\%) (Seo et al., 2016). To improve the ability of the Jeju horse in racing, crossbreeding with the Thoroughbred horse was initiated. This led to the emergence of hybrid breeds, such as the Halla horse.

Halla horse is crossbreed between Jeju and Thoroughbred horses and is used for riding, racing and meat production. But, a little research has been conducted on Halla horses because of the perception of crossbreed and people's weighted interest toward Jeju horses. 
Therefore, molecular genetic studies are needed to establish and preserve the industrially valuable Halla horses. And it is essential to do genetic characterization to compared different horse populations for a knowledge about their genetic specificity can help us to do the best plan about their management and conservation (Berber et al., 2014).

This study was conducted to analyze the genetic background and polymorphism in various horse breeds (Jeju, Mongolian, and Thoroughbred horses) related to the origins of the Halla horse in Korea using 12 microsatellite (MS) markers.

\section{MATERIALS AND METHODS}

\section{Animals and DNA isolation}

For DNA analysis using microsatellites, 200 Halla horses and 84 Jeju horses in Korea, 36 Mongolian horses in Mongolia and 40 Thoroughbred horses (International Society for Animal Genetics, ISAG Comparison test DNA sample) were used. Excluding the Thoroughbred horses, genomic DNA was extracted from hair roots of 320 horses using the methods described for QuickGene DNA tissue kits (FUJIFILM, Tokyo, Japan), and the concentration and purity of the isolated genomic DNA were evaluated using an ND-1000 UV-Vis Spectrophotometer (NanoDrop Technologies, Wilmington, USA), and used for analysis.

\section{Information on microsatellite (MS) markers}

The genetic diversity of the horse breeds was analyzed using 12 MS markers recommended by the ISAG: AHT4, AHT5, ASB2, ASB17 (Binns et al., 1995); ASB23 (Irvin et al., 1998); HMS2, HMS3, HMS6, HMS7 (Guékin et al., 1994); HTG4 (Ellegren et al., 1992); HTG10 (Marklund et al., 1994) and VHL20 (van Haeringen et al., 1994).

\section{Short Tandom Repeat (STR) Genotyping}

Multiplex PCR amplification was conducted using Equine Genotypes Panel 1.1 Kit (Thermo Fisher Scientific, USA) and PCR was performed using GeneAmp PCR System 9700 (Applied Biosystems, CA, USA). STR genotyping was performed using an Genetic Analyzer 3130xl (Applied Biosystems, CA, USA) following previously described methods (Seo et al., 2016).

\section{Statistical analysis of data}

Data from genotyping were analyzed using Cervus V 3.0 (Marchall et al., 1998) and MS Excel toolkit version 3.1 (Park, 2001) to calculate allele frequencies at each locus for each population, $\mathrm{H}_{\text {exp }}, \mathrm{H}_{\mathrm{obs}}$, and PIC values. Inbreeding-like effects within subpopulations (genetic distance $\left[\mathrm{F}_{s t}\right]$ ), among subpopulations (within inbreeding $\left[\mathrm{F}_{i s}\right]$ ), and within the entire population (total inbreeding $\left[\mathrm{F}_{\text {it }}\right]$ ) were analyzed by F-statistics (Wright, 1965). PCoA (Principal Coordinates Analysis) was conducted for the 12 MS markers using GenAlEx 6.4. Factorial correspondence analysis (FCA), which is a weighted PCoA method, was performed using the allele frequency data for the individuals of all 4 breeds and 12 MS markers using the GENETIX software (Belkhir, 2003; Tantia et al., 2006).

The genetic diversity among the populations was evaluated based on allele frequencies according to genetic distance $D_{A}$ (Nei et al., 1983) using the POPTREE2 (Takezaki et al., 2010). A phylogenetic tree was constructed from the distances using neighbor - joining method (Saitou and Nei 1987). Population structure was analyzed using STRUCTURE version 2.3.4 (Pritchard et al., 2000), the length of burn-in was set to 500 and the number of Markov chain Monte Carlo (MCMC) iteration was set to 1,000. On the basis of the value of $\Delta K$ (Evanno et al., 2005), determined using Structure Harvester (Earl, 2001), we estimated the optimal $\mathrm{K}$ value.

\section{RESULTS}

The $\mathrm{H}_{\text {exp }}, \mathrm{H}_{\text {obs }}$, PIC, and F-statistics values for the 4 breeds are summarized in Table 1. For the 12 MS markers, the allele number was confirmed to range from 6 (HMS6) to 17 (ASB17). The total allele number for the 12 markers was 120 , and the mean allele number was 10 . The $\mathrm{H}_{\mathrm{obs}}$ values ranged from 0.619 (HTG4) to 0.827 (HTG10). AHT4 showed the highest $\mathrm{H}_{\text {exp }}$ and PIC values of 0.858 and 0.840 , respectively, while HTG4 showed the lowest $\mathrm{H}_{\text {exp }}$ and PIC values of 0.652 and 0.609 , respectively.

$\mathrm{F}$-statistics were estimated in fixation indices as genetic differentiation $\left(\mathrm{F}_{s t}\right)$, global heterozygote deficit among the 4 horse breeds $\left(\mathrm{F}_{i t}\right)$, and heterozygote deficit within the breed/line $\left(\mathrm{F}_{i \mathrm{~s}}\right)$ among the 12 MS markers (Table 1). Among these markers, $F_{s t}$ ranged from 0.022 (ASB23) to 0.074(AHT5); the global heterozygote deficit among the 4 horse breeds $\left(F_{i t}\right)$ ranged from 0.010 (ASB17) to 0.086 
Table 1.The statistical analysis of heterozygosity $\left(\mathrm{H}_{\mathrm{obs}}\right.$ and $\left.\mathrm{H}_{\text {exp }}\right)$, Polymorphism Information Content (PIC), and F-statistics value using selected 12 microsatellite markers

\begin{tabular}{lccccccc}
\hline Locus & No. of allele & $\mathrm{H}_{\text {obs }}$ & $\mathrm{H}_{\text {exp }}$ & $\mathrm{PIC}$ & $\mathrm{F}_{s t}(\theta)$ & $\mathrm{F}_{i t}(\mathrm{~F})$ & 0.078 \\
\hline AHT4 & 9 & 0.816 & 0.858 & 0.840 & 0.050 & 0.029 \\
AHT5 & 7 & 0.807 & 0.829 & 0.804 & 0.074 & 0.086 & 0.013 \\
ASB2 & 13 & 0.819 & 0.826 & 0.803 & 0.052 & 0.014 & -0.040 \\
ASB17 & 17 & 0.794 & 0.804 & 0.783 & 0.032 & 0.010 & -0.022 \\
ASB23 & 12 & 0.769 & 0.818 & 0.794 & 0.022 & 0.075 \\
HMS2 & 9 & 0.730 & 0.793 & 0.762 & 0.072 & 0.119 & 0.054 \\
HMS3 & 10 & 0.725 & 0.814 & 0.787 & 0.060 & 0.128 & 0.051 \\
HMS6 & 6 & 0.750 & 0.763 & 0.724 & 0.037 & 0.023 & -0.014 \\
HMS7 & 9 & 0.739 & 0.755 & 0.720 & 0.052 & 0.028 & -0.025 \\
HTG4 & 7 & 0.619 & 0.652 & 0.609 & 0.050 & 0.060 & 0.011 \\
HTG10 & 12 & 0.827 & 0.839 & 0.819 & 0.039 & 0.055 & 0.017 \\
VHL20 & 9 & 0.808 & 0.831 & 0.810 & 0.046 & 0.028 & -0.019 \\
Mean & 10 & 0.767 & 0.799 & 0.771 & 0.049 & 0.059
\end{tabular}

$\mathrm{H}_{\mathrm{obs}}$ : Observed heterozygosity, $\mathrm{H}_{\mathrm{exp}}$ : Expected heterozygosity, PIC: Polymorphic information content, $\mathrm{F}_{s t}$ : Genetic distance, $\mathrm{F}_{i t}:$ Total inbreeding, $\mathrm{F}_{i s}$ : Within inbreeding.

Table 2.The statistical analysis of heterozygosity $\left(\mathrm{H}_{\mathrm{obs}}\right.$ and $\left.\mathrm{H}_{\mathrm{exp}}\right)$ and polymorphism information content (PIC) observed across 12 microsatellite loci for each breeds.

\begin{tabular}{lccc}
\hline \multicolumn{1}{c}{ Breeds } & $\mathrm{H}_{\text {obs }}$ & $\mathrm{H}_{\text {exp }}$ & $\mathrm{PIC}$ \\
\hline Halla horses & 0.782 & 0.786 & 0.756 \\
Jeju horses & 0.749 & 0.738 & 0.698 \\
Mongolian horses & 0.771 & 0.801 & 0.764 \\
Thoroughbred horses & 0.722 & 0.758 & 0.712 \\
\hline
\end{tabular}

$\mathrm{H}_{\text {exp }}$ :Expected heterozygosity, $\mathrm{H}_{\text {obs }}:$ Observed heterozygosity, PIC: Polymorphic information content.

(AHT5), and the heterozygote deficit within the breed/ line $\left(\mathrm{F}_{\text {is }}\right)$ ranged from-0.040 (ASB2) to 0.072 (HMS3). The breed statistics generated by the 12 MS markers in 4 horse breeds are shown in Table 2. The mean $\mathrm{H}_{\mathrm{obs}}$ ranged from 0.722 in the Thoroughbred horse to 0.782 in Halla horse. Average $\mathrm{H}_{\text {exp }}$ ranged from 0.738 in the Jeju horse to 0.801 in the Mongolian horse.

Fig. 1 illustrates the population relationships based on PCoA using individual multilocus genotypes of the $12 \mathrm{MS}$ markers. The contribution to the variance of the principal components exceeded $60 \%$, including the three components. The first and second components contributed $27.72 \%$ and $17.30 \%$, respectively, and the third component contributed $16.29 \%$. Clearly, based on the first component, Thoroughbred and Jeju horses were confirmed to be separated. Furthermore, Halla horse was confirmed to be between Thoroughbred and Jeju horses. The neighbornetwork analysis of the 4 breeds confirmed the Factorial correspondence analysis (FCA) results as the horse breeds

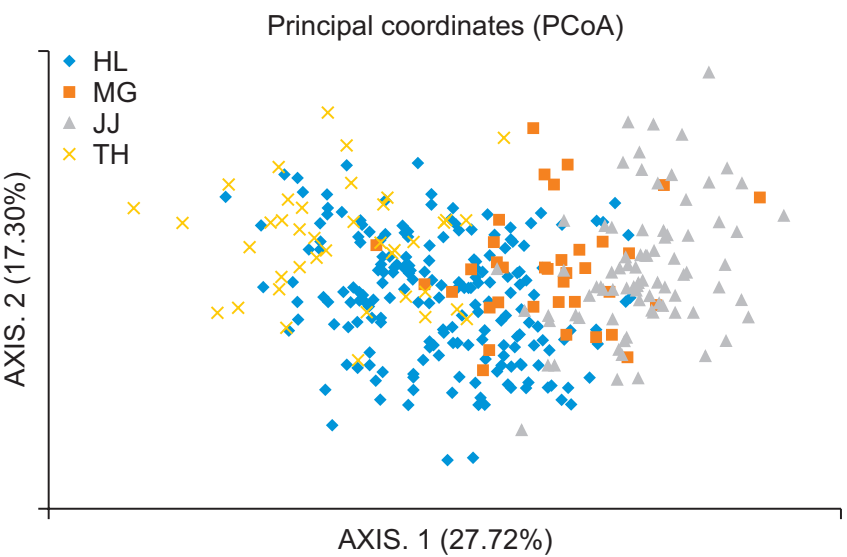

Fig. 1. PCoA of allele frequencies from 12 microsatellite markers typed in 4 breeds using GenAIEx. HL, Halla horse; MG, Mongolian horse; JJ, Jeju horse; TH, Thoroughbred horse.

segregated in a similar pattern of Fig. 1 (Fig. 2). Based on the results of neighbor - joining analysis, Halla horse was between Thoroughbred and Jeju horses and separated the Thoroughbred and Jeju horses (Fig. 3).

We applied the STRUCTURE program (Pritchard et al., 2000) to estimate the relationship between the Halla horse and putatively related breeds (Fig. 4, Supplementaty Table 1). On the basis of the $\Delta \mathrm{K}$ value of 11.764 obtained using Structure Harvester, we estimated the optimal $\mathrm{K}$ value to be 3 , corresponding to 4 breeds. These populations resulted in the separation of the 3 breeds and as shown above, Thoroughbred and Jeju horses were confirmed to be separated. 


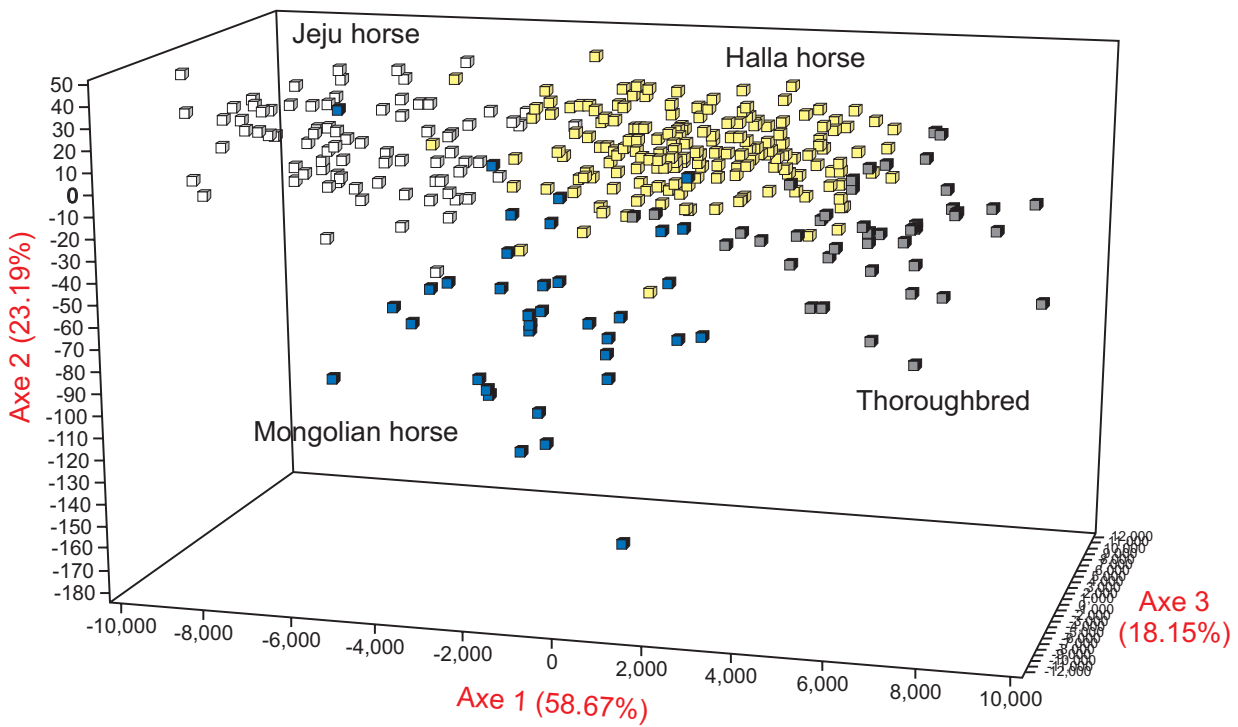

Fig. 2. Correspondence analysis of allele frequencies from 12 microsatellite markers typed in 4 breeds using Genetix405. Yellow, Halla horse; White, Jeju horse: Blue, Mongolian horse; Gray, Thoroughbred horse.

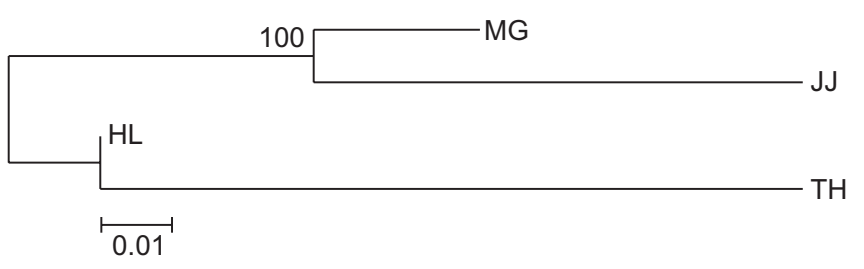

Fig. 3. Phylogenetic tree for 4 breeds on 12 microsatellite markers using neighbor - joining method. HL, Halla horse; MG, Mongolian horse; JJ, Jeju horse; TH, Thoroughbred horse.

\section{DISCUSSION}

This study aimed to analyses the genetic diversity and population structure through 12 microsatellite (MS) markers for Halla and putatively related 3 breeds. The allele number was confirmed to range from 6 (HMS6) to 17 (ASB17) and the $\mathrm{H}_{\mathrm{obs}}$ values ranged from 0.619 (HTG4) to 0.827 (HTG10). AHT4 showed the highest $\mathrm{H}_{\exp }$ and PIC values of 0.858 and 0.840 , respectively, while HTG 4 showed the lowest $\mathrm{H}_{\exp }$ and PIC values of 0.652 and 0.609 , respectively. In a previous study by Seo et al. (2016) the genetic diversity of 3,880 Halla horses were evaluated using MS markers (Seo et al., 2016). The allele number was reported to be the same or higher for all markers than in this study, excluding the HMS7 marker. These results were considered to be due to the population size. However, in this study, the observation that the HMS7 marker showed a higher allele frequency than in the previous study was considered to be due to the other populations (Jeju, Mongolian, and Thoroughbred horses). Botstein et al. (1980) reported that for animal traceability, PIC $>0.5$ and $\mathrm{H}_{\exp }>$
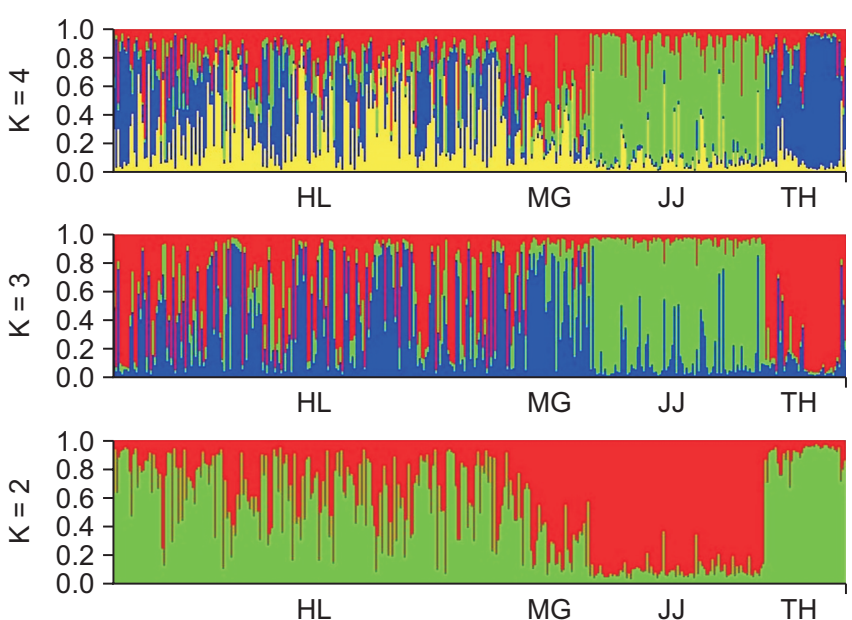

Fig. 4. Population structure of the analyzed 4 breeds using modelbased clustering method implemented in STRUCTURE at $\mathrm{K}=2,3$ and 4 . Each column represents the proportion in which an individual belongs to a different coloured cluster. The optimal $K$ value was estimated to be $3(\Delta K=11.764)$, as determined by Structure Harvester. HL, Halla horse; MG, Mongolian horse; JJ, Jeju horse; $\mathrm{TH}$, Thoroughbred horse.

0.6 are the most reasonable informative loci for application in genetics. These $12 \mathrm{MS}$ markers used in this study showed high polymorphism in the 4 breeds.

Fig. 1 illustrates the population relationships based on PCoA using individual multilocus genotypes of the 12 MS markers. Clearly, based on the first component, Thoroughbred and Jeju horses were confirmed to be separated. Furthermore, Halla horse was confirmed to be between Thoroughbred and Jeju horses. The neighbornetwork analysis of the 4 breeds confirmed the Factorial 
correspondence analysis (FCA) results as the horse breeds segregated in a similar pattern of Fig. 1 (Fig. 2). Based on the results of neighbor - joining analysis, Halla horse was between Thoroughbred and Jeju horses and separated the Thoroughbred and Jeju horses (Fig. 3). These results are attributed to the fact that Halla horse is crossbreeding between Thoroughbred and Jeju horses. It is also supposed that Halla horse is an original breed and with a specific genetic structure comfort its industrial interest. Furthermore, the phylogenetic tree showed that Mongolian horse and Jeju horse were closely related species (Fig. 3). It is supported by the historical fact that Jeju horses were mixed origin in their maternal lineage (Lee et al., 2010).

To further investigate the relationship between the Halla horse and putatively related breeds, we analyzed the STRUCTURE program (Fig. 4). These populations resulted in the separation of the 3 breeds and as shown above, Thoroughbred and Jeju horses were confirmed to be separated. Halla horses were between Thoroughbred and Jeju horses, tend to more influenced by Thoroughbred horses and similar to Mongolian horses.

In this study, we analyzed the genetic variation and relationship of Halla and putatively related 3 breeds (Jeju, Mongolian and Thoroughbred horses) using 12 MS marker, and we support the view that Halla horses originated between Thoroughbred and Jeju horses.

Therefore, these results could be considered for use as the basic genetic breed relationships resource among the horse breeds (Jeju, Mongolian, and Thoroughbred horses) related to the origins of the Halla horse. And it also obtained in the present study contribute to establishing the cross combination of the Halla horse and will provide important to support the future monitoring of population diversity. Futhermore, since the MS markers in this study are highly polymorphic, they can also be applied for paternity testing.

\section{CONCLUSION}

In conclusion, we analyses the genetic diversity and population structure through 12 microsatellite (MS) markers for Halla and putatively related 3 breeds (Jeju, Mongolian and Thoroughbred horses). As a results, Neighborjoining tree and STRUCTURE analysis showed that Halla horses were between Thoroughbred and Jeju horses, tend to more influenced by Thoroughbred horses. Therefore, we support the view that Halla horses originated between
Thoroughbred and Jeju horses.

\section{CONFLICTS OF INTEREST}

No potential conflict of interest relevant to this article was reported.

\section{ETHICS APPROVAL}

The study was approved by the Hankyong National University Animal Ethics Committee (No.2015-4).

\section{AUTHOR CONTRIBUTIONS}

Conceptualization: Hong Sik Kong

Data curation: Ji Su Jung, Gwang Hyeon Lee,

Yesong Kim, Je Hyun An, Ji Hye Yun

Formal analysis: Ji Su Jung, Jiyeon Seong

Funding acquisition: Hong Sik Kong

Investigation: Ji Su Jung, Jiyeon Seong

Methodology: Ji Su Jung, Je Hyun An, Ji Hye Yun

Project administration: Hong Sik Kong

Resources: Hong Sik Kong

Software: Gwang Hyeon Lee, Yesong Kim

Supervision: Hong Sik Kong

Validation: Hong Sik Kong, Jiyeon Seong

Visualization: Ji Su Jung

Writing - original draft: Ji Su Jung, Jiyeon Seong

Writing - review \& editing: Hong Sik Kong, Jiyeon Seong

\section{AUTHOR'S POSITION AND ORCID NO.}

JS Jung, MS,

https://orcid.org/0000-0001-8776-1061

J Seong, PhD, https://orcid.org/0000-0003-0956-995X

GH Lee, Doctor's Course, https://orcid.org/0000-0002-6598-8430

Y Kim, Doctor's Course, https://orcid.org/0000-0001-8459-0519

JH An, Masters's Course, https://orcid.org/0000-0001-5253-6550

JH Yun, Masters's Course, https://orcid.org/0000-0002-4558-2337

HS Kong, Professor, https://orcid.org/0000-0003-1144-016X 


\section{SUPPLEMENTARY MATERIALS}

Supplementary material can be found via https://doi.12750/ JARB.36.2.76.

\section{REFERENCES}

Belkhir K, Borsa P, Chikhi L, Raufaste N. et al. 2003. GENETIX version 4.04.

Berber N, Gaouar S, Leroy G, Kdidi S, Tabet Aouel N, Saïdi Mehtar N. 2014. Molecular characterization and differentiation of five horse breeds raised in Algeria using polymorphic microsatellite markers. J. Anim. Breed. Genet. 131:387-394.

Binns MM, Holmes NG, Holliman A, Scott AM. 1995. The identification of polymorphic microsatellite loci in the horse and their use in thoroughbred parentage testing. Br. Vet. J. 151:915.

Botstein D, White RL, Skolnick M, Davis RW. 1980. Construction of a genetic linkage map in man using restriction fragment length polymorphisms. Am. J. Hum. Genet. 32:314331.

Earl DA. 2001. Structure harvester v0.6.1. http://taylor0.biology.ucla.edu/struct_harvest/ [accessed on December 20, 2010].

Ellegren H, Johansson M, Sandberg K, Andersson L. 1992. Cloning of highly polymorphic microsatellites in the horse. Anim. Genet. 23:133-142.

Evanno G, Regnaut S, Goudet J. 2005. Detecting the number of clusters of individuals using the software STRUCTURE: a simulation study. Mol. Ecol. 14:2611-2620.

Guérin G, Bertaud M, Amigues Y. 1994. Characterization of seven new horse microsatellites: HMS1, HMS2, HMS3, HMS5, HMS6, HMS7 and HMS8. Anim. Genet. 25:62.

Irvin Z, Giffard J, Brandon R, Breen M, Bell K. 1998. Equine dinucleotide repeat polymorphisms at loci ASB 21, 23, 25 and 37-43. Anim. Genet. 29:67.
Lee JE, Shin JH, Yun YM, Lee KK, Lee H, Kweon OK, Yun YS, Suh JG, Shin NS, Seong JK. 2010. Genetic polymorphism of Jeju horses by microsatellite DNA markers in Korea. Lab. Anim. Res. 26:219-221.

Marklund S, Ellegren H, Eriksson S, Sandberg K, Andersson L. 1994. Parentage testing and linkage analysis in the horse using a set of highly polymorphic microsatellites. Anim. Genet. 25:19-23.

Marshall TC, Slate J, Kruuk LE, Pemberton JM. 1998. Statistical confidence for likelihood-based paternity inference in natural populations. Mol. Ecol. 7:639-655.

Nei M, Tajima F, Tateno Y. 1983. Accuracy of estimated phylogenetic trees from molecular data. II. Gene frequency data. J. Mol. Evol. 19:153-170.

Park SDE. 2001. The Excel microsatellite toolkit (version 3.1).

Pritchard JK, Stephens M, Donnelly P. 2000. Inference of population structure using multilocus genotype data. Genetics 155:945-959.

Saitou N and Nei M. 1987. The neighbor-joining method: a new method for reconstructing phylogenetic trees. Mol. Biol. Evol. 4:406-425.

Seo JH, Park KD, Lee HK, Kong HS. 2016. Genetic diversity of Halla horses using microsatellite markers. J. Anim. Sci. Technol. 58:40.

Takezaki N, Nei M, Tamura K. 2010. POPTREE2: software for constructing population trees from allele frequency data and computing other population statistics with Windows interface. Mol. Biol. Evol. 27:747-752.

Tantia MS, Vijh RK, Mishra B, Kumar STB, Arora R. 2006. Multilocus genotyping to study population structure in three buffalo populations of India. Asian-Aust. J. Anim. Sci. 19:10711078.

van Haeringen H, Bowling AT, Stott ML, Lenstra JA, Zwaagstra KA. 1994. A highly polymorphic horse microsatellite locus: VHL20. Anim. Genet. 25:207.

Wright DJ. 1965. General multiplicity theory. Proc. Lond. Math. Soc. s3-15:269-288. 